\title{
BRD4 regulates Nanog expression in mouse embryonic stem cells and preimplantation embryos
}

\author{
W Liu', P Stein ${ }^{2}, X$ Cheng $^{3}$, W Yang ${ }^{4}$, N-Y Shao ${ }^{5}$, EE Morrisey ${ }^{4}$, RM Schultz ${ }^{2}$ and J You ${ }^{*, 1}$
}

Bromodomain-containing protein 4 (BRD4) is an important epigenetic reader implicated in the pathogenesis of a number of different cancers and other diseases. Brd4-null mouse embryos die shortly after implantation and are compromised in their ability to maintain the inner cell mass, which gives rise to embryonic stem cells (ESCs). Here we report that BRD4 regulates expression of the pluripotency factor Nanog in mouse ESCs and preimplantation embryos, as well as in human ESCs and embryonic cancer stem cells. Inhibition of BRD4 function using a chemical inhibitor, small interfering RNAs, or a dominantnegative approach suppresses Nanog expression, and abolishes the self-renewal ability of ESCs. We also find that BRD4 associates with BRG1 (brahma-related gene 1, aka Smarca4 (SWI/SNF-related, matrix-associated, actin-dependent regulator of chromatin, subfamily a, member 4)), a key regulator of ESC self-renewal and pluripotency, in the Nanog regulatory regions to regulate Nanog expression. Our study identifies Nanog as a novel BRD4 target gene, providing new insights for the biological function of BRD4 in stem cells and mouse embryos. Knowledge gained from these non-cancerous systems will facilitate future investigations of how Brd4 dysfunction leads to cancers.

Cell Death and Differentiation (2014) 21, 1950-1960; doi:10.1038/cdd.2014.124; published online 22 August 2014

Bromodomain-containing protein 4 (BRD4) belongs to the bromodomain and extraterminal (BET) protein family. ${ }^{1}$ BRD4 functions as an epigenetic reader by binding to acetylated histones on chromatin through its two bromodomains, and has a central role in transcriptional regulation, cellular growth control and cell cycle progression. ${ }^{2}$ BRD4 supports transcriptional activation by actively recruiting the positive transcription elongation factor $b$, mediators and several other transcriptional activators. ${ }^{2,3}$

BRD4 is implicated in the pathogenesis of a number of cancers and other diseases. ${ }^{3-8}$ In some cancers, BRD4 regulates expression of $c-M y c$ and other oncogenes. ${ }^{3,4}$ It also selectively binds to the 'super-enhancers' of tumor oncogenes, which are large clusters of enhancers that control expression of these genes. ${ }^{3,9}$ Although these recent studies have shed light on the gene-specific activity of BRD4, how alterations in BRD4 function contribute to the development of cancers and other diseases is not well understood. This lack of knowledge reflects the need to better understand the normal function of BRD4 in noncancerous cells, as most of the previous studies of BRD4 function were performed in cancer cells.

In Brd4 knockout mice, the homozygous embryos die shortly after implantation. ${ }^{10}$ Cells derived from the inner cell mass (ICM) of these homozygous embryos are completely degenerated, although the rest of the embryo appears morphologically normal. ${ }^{10}$ These observations suggest that Brd4 is required for the development and/or maintenance of the $\mathrm{ICM},{ }^{10}$ which gives rise to embryonic stem cells (ESCs) in culture. ESCs with homozygous Brd4 deletion are nonviable, ${ }^{11}$ further supporting the idea that Brd4 is important for ESC proliferation and maintenance. We therefore examined Brd4 function in ESCs and preimplantation embryos.

ESCs derived from the ICM of day 3.5 mouse blastocysts are characterized by their pluripotency and self-renewal capacity. The transcription factors NANOG, POU5F1 (OCT4) and SOX2 are the core regulatory factors of selfrenewal and pluripotency that maintain ESC propagation in an undifferentiated state. ${ }^{12}$ Expression of these pluripotency regulators is tightly controlled through a transcriptional circuitry consisting of auto-regulatory feedback loops. ${ }^{13}$ Nanog has a particularly important role in establishing ESC ground state pluripotency. ${ }^{14,15}$ Nanog null ESCs are prone to differentiate, ${ }^{16}$ and knockdown of Nanog in mouse ESCs causes loss of self-renewal and induction of trophectoderm and primitive endoderm differentiation. ${ }^{17}$ Pou5f1 and Nanog are also key regulators of early mouse embryo development. ${ }^{14,15,18,19}$ Although these studies have shed

\footnotetext{
${ }^{1}$ Department of Microbiology, University of Pennsylvania, Perelman School of Medicine, Philadelphia, PA, USA; ${ }^{2}$ Department of Biology, University of Pennsylvania, Philadelphia, PA, USA; ${ }^{3}$ State Key Laboratory of Cell Biology, Institute of Biochemistry and Cell Biology, Shanghai Institutes for Biological Sciences, Chinese Academy of Sciences, Shanghai, China; ${ }^{4}$ Institute for Regenerative Medicine, University of Pennsylvania, Philadelphia, PA, USA and ${ }^{5}$ Fishberg Department of Neuroscience and Friedman Brain Institute, Icahn School of Medicine at Mount Sinai, New York, NY, USA

${ }^{*}$ Corresponding author: J You, Department of Microbiology, University of Pennsylvania, Perelman School of Medicine, 3610 Hamilton Walk, 201C Johnson Pavilion, Philadelphia, PA 19104, USA. Tel: +1 215573 6781; Fax: +1 215898 9557; E-mail: jianyou@ mail.med.upenn.edu

Abbreviations: Brd4, bromodomain-containing protein 4; ICM, inner cell mass; ESCs, embryonic stem cells; BET, bromodomain and extraterminal protein; LIF, leukemia inhibitory factor; BMP4, bone morphogenetic protein 4; AP, alkaline phosphatase; siRNAs, small interfering RNAs; BDI/II, double bromodomains; ChIP, chromatin immunoprecipitation; BRG1, brahma-related gene 1, aka Smarca4 (SWI/SNF-related, matrix-associated, actin-dependent regulator of chromatin, subfamily a, member 4); ChIP-reChIP, sequential ChIP analysis; H3K27me3, trimethylation of Lys27 of histone 3; FBS, fetal calf serum; GV, germinal vesicle; MII, metaphase II; PVP, polyvinylpyrrolidone; KSOM + AA, KSOM medium containing amino acids; RA, retinoic acid

Received 06.6.14; revised 10.7.14; accepted 13.7.14; Edited by D Vaux; published online 22.8.14
} 
light on how transcription factor networks regulate ESC pluripotency, further studies are necessary to understand fully the molecular mechanisms that regulate these core factors in preimplantation embryos and ESCs.

We report that BRD4 regulates Nanog expression in ESCs and preimplantation embryos. Inhibiting BRD4 function abolishes Nanog expression and abrogates ESC maintenance. Furthermore, we find that BRD4 associates with the chromatin-remodeling protein BRG1 (brahma-related gene 1, aka Smarca4 (SWI/SNF-related, matrix-associated, actindependent regulator of chromatin, subfamily a, member 4)) and binds to the Nanog regulatory regions, a finding that likely underlies the ability of BRD4 to regulate Nanog expression in ESCs.

\section{Results}

Brd4 is downregulated during mouse ESC differentiation. To explore Brd4 function in ESCs, we analyzed BRD4 protein levels in two different mouse ESC lines, TL1 and R1, before and after differentiation induced by withdrawal of leukemia inhibitory factor (LIF)/bone morphogenetic protein 4 (BMP4) and addition of retinoic acid (RA). Interestingly, Brd4 protein levels decreased markedly in both ESC lines when they were induced to differentiate (Figure 1). The reduction in the amount of BRD4 protein paralleled the decrease in the amount of NANOG, POU5F1 and SOX2 protein during ESC differentiation. This result suggests that BRD4 may have a more important role in undifferentiated ESCs than in differentiated cells.

BRD4 inhibitor JQ1 $(+)$ impairs ESC maintenance. To examine further BRD4 function in undifferentiated ESCs, we tested how inhibiting BRD4 affected ESC growth and maintenance. JQ1 $(+)$, which is a BET protein inhibitor, displays the highest affinity for BRD4, and prevents BRD4 binding to acetylated histones. ${ }^{4,6}$ TL1 and R1 ESCs were treated with $\mathrm{JQ1}(+)$ and the inactive stereoisomer, JQ1(-),

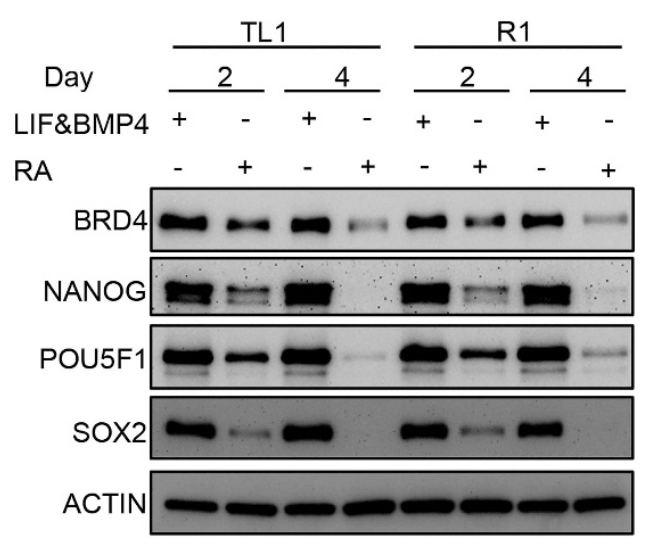

Figure 1 BRD4 is downregulated during ESC differentiation. TL1 and R1 ESCs were cultured in the presence of $1000 \mathrm{U} / \mathrm{ml} \mathrm{LIF}$ and $10 \mathrm{ng} / \mathrm{ml}$ human BMP4 or in the presence of $1 \mu \mathrm{M}$ RA without LIF and BMP4 for 48 or $96 \mathrm{~h}$ to induce differentiation. Whole-cell lysates were immunoblotted using the indicated antibodies. ACTIN was used as a loading control. This experiment was performed at least three times with similar results to test their effect on ESC maintenance. Compared with untreated or JQ1(-)-treated cells, JQ1(+)-treated ESCs displayed an abnormal morphology, and also generated very few ESC colonies with high alkaline phosphatase (AP) activity, a marker for undifferentiated ESCs (Figures 2a and b). This observation suggests that JQ1 $(+)$ inhibits ESC maintenance and induces cell differentiation.

We then analyzed the effect of JQ1 $(+)$ on expression of the key stem cell pluripotency regulators, Nanog, Pou5f1 and Sox2. Upon JQ1 $(+)$ treatment, the NANOG protein level was reduced to $25 \% \pm 6 \%$ in TL1 cells and $20 \% \pm 3 \%$ in $\mathrm{R} 1$ cells when compared with untreated cells, whereas the level in JQ1(-)-treated cells remained similar to untreated cells (Figure 2c and Supplementary Figure S1A). On the other hand, a very moderate reduction was observed for POU5F1 $(87 \% \pm 7 \%$ in TL1, $86 \% \pm 8 \%$ in R1) and SOX2 $(74 \% \pm 13 \%$ in TL1, $76 \% \pm 8 \%$ in R1) in JQ1(+)-treated cells when compared with untreated (Figure $2 \mathrm{C}$ and Supplementary Figure S1A). The protein level of $\mathrm{c}-\mathrm{MYC}$, a known BRD4 target in cancer cells, was also not markedly reduced by JQ1 (+) treatment $(72 \% \pm 3 \%$ in TL1, 85\% $\pm 5 \%$ in R1). $c-M y c$ is dispensable in ESCs and in mouse embryos before $\mathrm{E} 10.5,{ }^{20}$ suggesting that BRD4 regulates genes other than $c-M y c$ in ESCs and during early mouse development. We therefore focused on the core ESC pluripotency regulators, Nanog, Pou5f1 and Sox2. Importantly, JQ1(+) treatment of human ESCs also led to a clear reduction of NANOG protein level (Supplementary Figure S1B).

JQ1 (+) inhibition of Nanog expression was confirmed by quantitative reverse transcription and real-time PCR (qRTPCR) in both TL1 and R1 cells, in which JQ1 $(+)$ had no marked effect on Pou5f1 and Sox2 expression (Figure 2d and Supplementary Figure S1C). In addition, JQ1(-) treatment did not cause clear changes in the expression of any of these genes (Figure 2d and Supplementary Figure S1C).

To confirm that JQ1 ( + ) promotes ESC differentiation, qRTPCR was performed to analyze expression of several early differentiation markers in the untreated cells and cells treated with either JQ1 (+ ) or JQ1 ( - ). JQ1 (+ ) treatment resulted in a selective upregulation of trophectodermal makers $C d x 2$ and Hand1 in both TL1 and R1 cells (Figure $2 e$ and Supplementary Figure S1D), a finding consistent with the observation that Nanog knockdown leads to mouse ESCs differentiating into the extraembryonic endoderm/trophectoderm lineages. ${ }^{15,17}$ Induction of these differentiation markers by JQ1 $(+)$ also indicated that BRD4 inhibition was targeting expression of specific genes, that is, it did not result in a global inhibition of transcription. On the other hand, it is important to note that the induction of the differentiation markers shown in Figure $2 \mathrm{e}$ and Supplementary Figure S1D could also result from the JQ1 $(+)$ inhibition of other BET proteins, which is addressed in the small interfering RNA (siRNA) experiments (see below). Collectively, these data demonstrate that JQ1 $(+)$ impairs ESC maintenance, leading to ES cell differentiation.

Brd4 knockdown and a BRD4 dominant-negative inhibitor abolish Nanog expression and ESC maintenance. To rule out effects of $\mathrm{JQ1}(+)$ on other BET proteins and to confirm the functional requirement for Brd4 in ESCs, we 
a TL1 ESCs

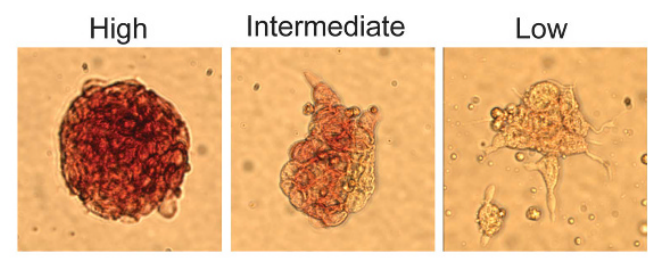

c TL1 ESCs

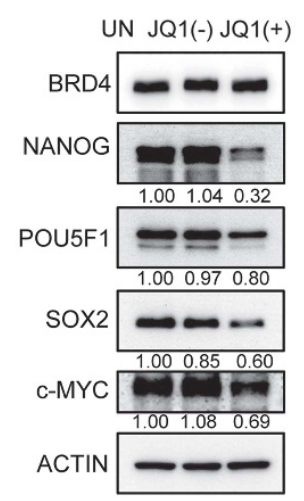

e TL1 ESCs

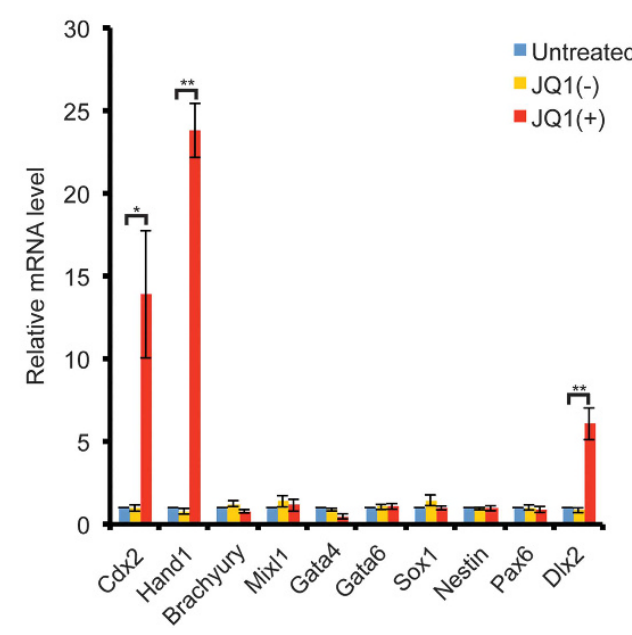

d TL1 ESCs

Untreated

JQ1(-) b

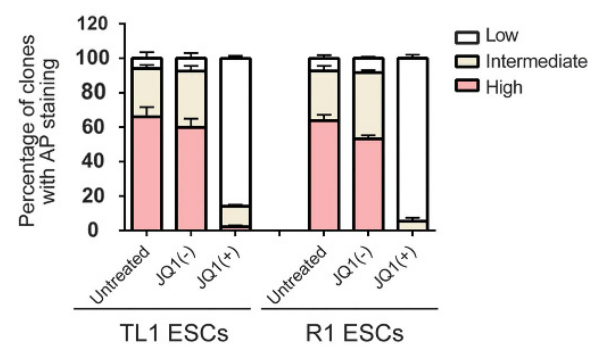

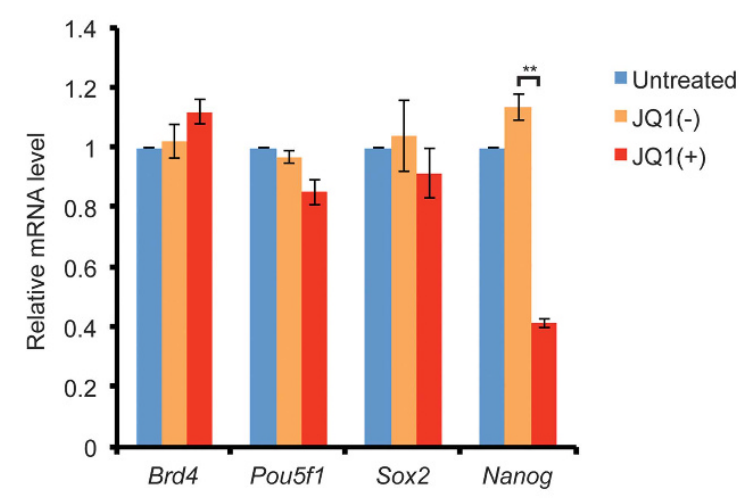

Figure $2 \mathrm{JQ1}(+)$ impairs ESC maintenance. (a) Representative images of ESC colonies showing high, intermediate or low AP activity. (b) TL1 and R1 ESCs were either untreated, treated with $300 \mathrm{nM}$ of JQ1 $(-)$ or JQ1 $(+)$ for $48 \mathrm{~h}$. The percentage of ESC colonies with high, intermediate and low AP activity was quantified from $>100$ colonies. (c) TL1 ESCs were prepared as in b. Whole-cell lysates were immunoblotted with the indicated antibodies. ACTIN was used as a loading control. UN, untreated cells. This experiment was performed at least three times with similar results. (d and e) TL1 ESCs were treated as in b for $24 \mathrm{~h}$ (in $\mathbf{d}$ ) or $48 \mathrm{~h}$ (in $\mathbf{e}$ ). The mRNA levels were measured by RT-qPCR and normalized to Gapdh mRNA levels. The mRNA levels in untreated cells were set as 1. All error bars represent S.E.M. from three independent experiments. ${ }^{*} P<0.05,{ }^{* *} P<0.01$

knocked down Brd4 transcripts using two different siRNAs. As complete loss of $B r d 4$ prevents ESC proliferation, ${ }^{11}$ we suppressed Brd4 expression using a siRNA concentration that partially knocked down Brd4 transcripts and proteins in TL1 and R1 cells (Figures $3 a-c$, Supplementary Figures S2A-S2C). We also confirmed that partial Brd4 knockdown induced by these siRNAs only moderately inhibited proliferation of either TL1 or R1 cells (Supplementary Figure S3).
Consistent with the observation in JQ1(+)-treated cells, Brd4 knockdown greatly reduced NANOG protein $(41 \% \pm 6 \%$ in TL1, $65 \% \pm 3 \%$ in R1) or transcripts in ESCs, but there was very little decrease in the amount of POU5F1 protein $(93 \% \pm 4 \%$ in $\mathrm{TL} 1,94 \% \pm 2 \%$ in $\mathrm{R} 1)$ and SOX2 protein $(99 \% \pm 2 \%$ in $\mathrm{TL} 1,94 \% \pm 7 \%$ in $\mathrm{R} 1)$ or these transcripts in both ESC lines (Figures $3 a$ and b, Supplementary Figures S2A and S2B). In addition, Brd4 knockdown in TL1 and R1 
a TL1 ESCs

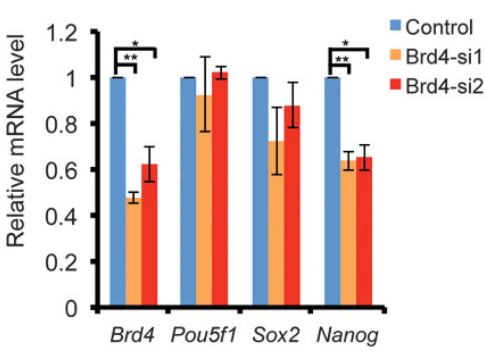

d TL1 ESCs

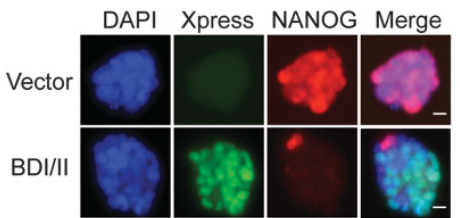

b TL1 ESCs

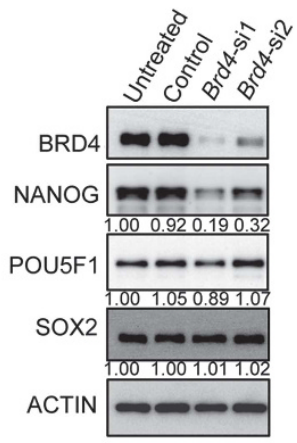

c R1 ESCs

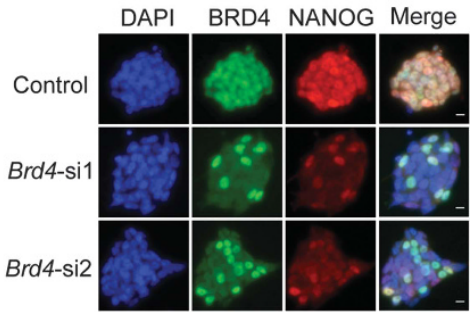

e TL1 ESCs

Percentage of cells with NANOG staining

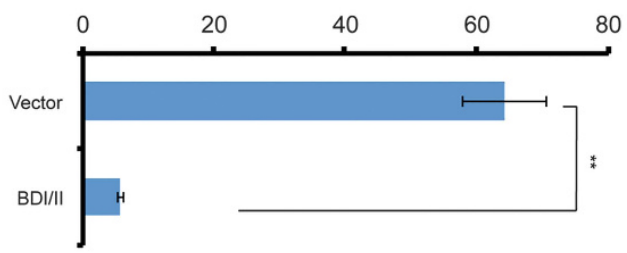

f TL1 ESCs
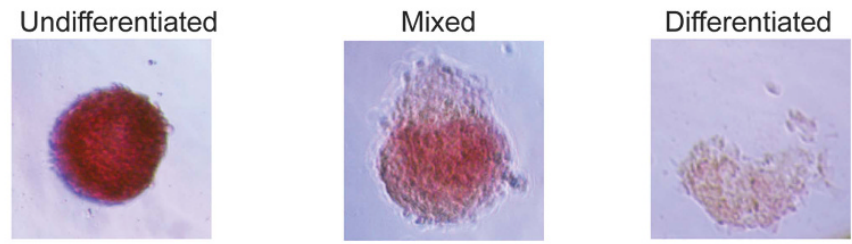

g

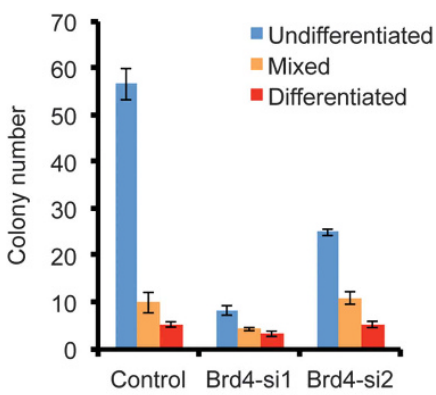

h TL1 ESCs

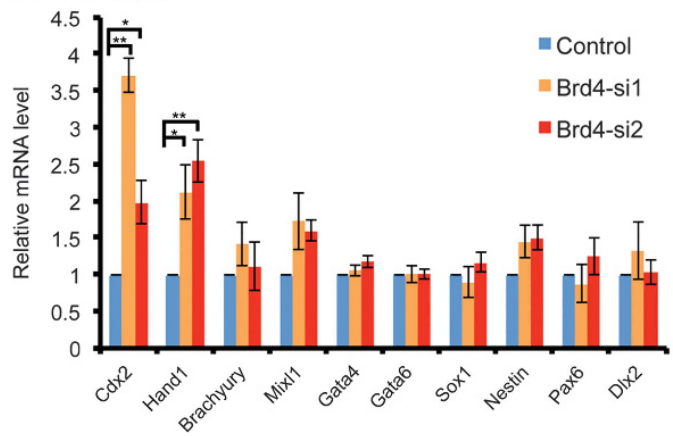

Figure 3 BRD4 inhibition abolishes Nanog expression in ESCs. (a) TL1 ESCs were transfected with control siRNA, Brd4 siRNA 1 or Brd4 siRNA 2. At 48-h posttransfection, the mRNA levels were measured by RT-qPCR and normalized to Gapdh mRNA levels. The mRNA levels in control cells were set as 1. (b) TL1 ESCs were either untreated or transfected as in a. At 48-h post-transfection, whole-cell lysates were extracted and immunoblotted with the indicated antibodies. Protein signal intensity was quantified using ImageJ. ACTIN was used as a loading control. This experiment was performed at least three times with similar results. (c) R1 ESCs were transfected as in (a). At 48-h post-transfection, the cells were co-stained with BRD4 and NANOG antibodies and counterstained with DAPI. Bar, $10 \mu \mathrm{m}$. (d and e) TL1 ESCs carrying a BDI/Iinducible expression cassette or an empty vector were treated with $0.1 \mu \mathrm{g} / \mathrm{ml}$ doxycycline for $24 \mathrm{~h}$ to induce BDI/ll expression. The cells were co-stained with Xpress and Nanog antibodies and counterstained with DAPI. Bar, $10 \mu \mathrm{m}$. The percentage of cells with positive Nanog signal was quantified from $>100$ cells. (f) Representative images of undifferentiated, mixed and differentiated TL1 ESC colonies. (g) TL1 ESCs were transfected as in $\mathbf{a}$. The number of colonies generated from 500 seeded cells was quantified at day 6 post-transfection. (h) TL1 ESCs were transfected as in a. At 72-h post-transfection, the mRNA levels were measured by RT-qPCR and normalized to Gapdh mRNA levels. The mRNA levels in control cells were set as 1. Values in (a), (e), (g) and (h) represent the average of three independent experiments with error bars indicating S.E.M. ${ }^{\star} P<0.05,{ }^{* \star} P<0.01$

cells grown in 2i/LIF condition also led to a clear reduction of NANOG protein (Supplementary Figure S2D).

We also performed microarray analysis to examine the global gene expression changes induced by Brd4 knockdown in mESCs (GEO accession number: GSE59142). As anticipated, we identified a large number of genes differentially regulated by Brd4 knockdown (Supplementary Figure S4). Among the core regulators of ESC pluripotency, Nanog was the only one inhibited by Brd4 knockdown whereas expression of Sox2 and Pou5f1 was unaltered by Brd4 knockdown. We also 
compared the differentially expressed genes identified in the Brd4 knockdown microarray analysis with the Nanog knockdown data (GEO accession number: GSE26520) ${ }^{21}$ and found a striking overlap between the lists of genes downregulated by Brd4 knockdown and Nanog knockdown $\left(P\right.$-value $\left.=5 \times 10^{-11}\right) \quad$ (Supplementary Figure S5 and Supplementary Table S1).

To detect specifically the reduction of NANOG protein in Brd4 knocked-down cells, we performed NANOG immunostaining in cells treated with either control or Brd4-targeting siRNAs. This approach showed that Nanog expression was specifically inhibited in Brd4-depleted TL1 and R1 cells, whereas neighboring BRD4-positive cells showed a normal level of NANOG protein (Figure $3 \mathrm{c}$ and Supplementary Figure $\mathrm{S} 2 \mathrm{C}$ ). In addition, Brd4 knockdown in these cells did not cause much of a decrease in SOX2 protein (Supplementary Figure S6), confirming that Brd4 knockdown cells were viable and that Brd4 knockdown caused specific inhibition of Nanog expression.

We also used a BRD4 dominant-negative inhibitor encoding the BRD4 double bromodomains (BDI/II) to confirm further BRD4's role in controlling Nanog expression in ESCs. We previously demonstrated that the BDI/II inhibitor binds to acetylated histones and competitively dissociates BRD4 from chromatin without affecting the closely related BRD2. ${ }^{22}$ Immunostaining analysis showed that Nanog expression was specifically inhibited in cells expressing the BDI/II molecule (Figures 3d and e).

As NANOG is important for maintaining the propagation of ESCs in an undifferentiated pluripotent state, ${ }^{12-17}$ we examined how Brd4 knockdown-induced Nanog repression affected ESC proliferation. For both TL1 and R1, the large majority of cells grew into undifferentiated ES colonies, in which all cells displayed a high level of AP activity (Figure 3f, undifferentiated). There was also a small number of ESC colonies that either contained a mixture of AP-positive and AP-negative cells (Figure $3 f$, mixed) or cells that were completely negative for AP activity (Figure 3f, differentiated). Compared with the control siRNA-treated cells, cells treated with either Brd4 siRNA showed a markedly reduced number of 'undifferentiated' colonies without drastically affecting the 'mixed' and 'differentiated' population (Figures $3 f$ and $g$, and Supplementary Figure S2E), indicating that Brd4 is crucial for maintaining the growth and/or seeding efficiency of undifferentiated ESC colonies. In addition, qRT-PCR analysis revealed that Brd4 knockdown in ESCs also resulted in a selective upregulation of $C d x 2$ and Hand1 (Figure $3 \mathrm{~h}$ and Supplementary Figure S2F). Collectively, the data show that Brd4 knockdown inhibited Nanog expression and prevented ESC proliferation in the undifferentiated stage. In line with the observation that BRD4 was markedly reduced during ES differentiation (Figure 1), the specific inhibition of undifferentiated cells by Brd4 knockdown suggested an important role of Brd4 in the maintenance of undifferentiated ESCs. Similar to JQ1 $(+)$ treatment, Brd4 knockdown showed little inhibitory effect on Pou5f1 and Sox2, consistent with BRD4 specifically regulating Nanog expression and that Brd4 inhibition does not result in a global inhibition of transcription.
Brd4 knockdown downregulates Nanog in preimplantation mouse embryos. During preimplantation development, Nanog mRNA is first detected at the four-cell stage, ${ }^{23}$ whereas NANOG protein is first detected at the morula stage. ${ }^{15}$ As JQ1 $(+)$ and Brd4 siRNAs decrease Nanog expression in ESCs, we asked whether BRD4 regulates Nanog expression during preimplantation development. We first analyzed the expression profile of Brd4 in different stages of preimplantation embryos (Supplementary Figure $\mathrm{S} 7 \mathrm{~A})$. BRD4 was observed at all stages of preimplantation development. A similar pattern of Brd4 expression in oocytes to two-cell stage embryos was observed in a previous study. ${ }^{24}$

To determine whether BRD4 regulates Nanog expression during preimplantation development, we performed $\mathrm{Brd} 4$ knockdown by microinjecting control or Brd4 siRNA into twocell mouse embryos. The embryos were then cultured to the late morula or blastocyst stages and stained for both BRD4 and NANOG proteins. In Brd4 siRNA-treated embryos, the amount of BRD4 protein was efficiently reduced in all blastomeres (Figure 4). Although the embryos microinjected with control siRNA showed a normal level of NANOG protein, nearly no NANOG signal was detected in blastomeres in which BRD4 was substantially reduced (Figure 4). In contrast, robust POU5F1 signals were detected in Brd4 siRNA-treated late morula stage embryos (Supplementary Figure S7B), confirming that these embryos are viable. Compared with control embryos, a slight reduction of POU5F1 signal was observed in Brd4 siRNA-treated embryos (Supplementary Figure S7B). This finding may reflect the marked repression of Nanog expression induced by Brd4 knockdown as Nanog null ICMs also demonstrate reduced level of POU5F1 staining. ${ }^{14}$ Interestingly, all the embryos, including those treated with Brd4 siRNA, developed to the blastocyst stage and appeared morphologically normal (Supplementary Figure S7C); there was, however, a delay in development of Brd4 knockdown embryos, which were smaller and had fewer ICM cells (Figure 4 and Supplementary Figures S7B and C). These results are consistent with a previously published study showing that Brd4 homozygous null embryos isolated at E3 were morphologically indistinguishable from wild-type blastocysts. ${ }^{10}$ After in vitro culture for 2 days, explants grown from Brd4 homozygous null embryos appeared smaller than wildtype embryos but otherwise showed normal morphology. ${ }^{10}$ Similarly, E4.5 Nanog null mouse embryos carried blastocysts containing reduced ICM cells than wild-type embryos. ${ }^{14,15}$ The inhibition of Nanog expression by Brd4 knockdown during early mouse embryo development was consistent with the observations made in ESCs (Figure 3 and Supplementary Figure S2). Collectively, these studies provide evidence that Brd4 regulates Nanog expression not only in ESCs but also in mouse embryos.

BRD4 occupies regulatory regions of the Nanog gene in ESCs. Both JQ1(+) and Brd4 siRNA inhibition of Brd4 expression reduced Nanog expression in ESCs, suggesting that BRD4 contributes to Nanog transcriptional regulation. To understand the molecular mechanism underlying BRD4's function, we investigated whether Nanog is a direct BRD4 target by performing chromatin immunoprecipitation (ChIP) 
a Late morula stage
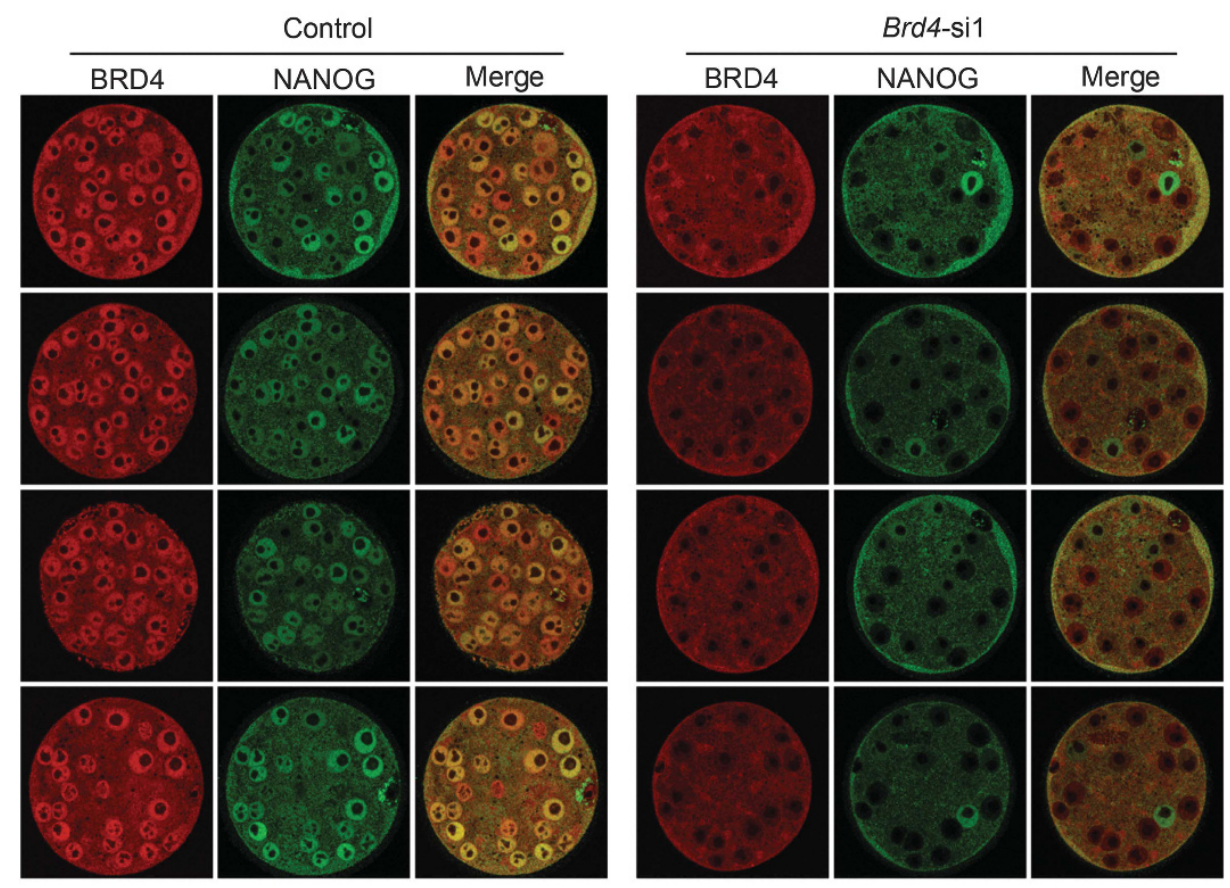

b Blastocyst stage
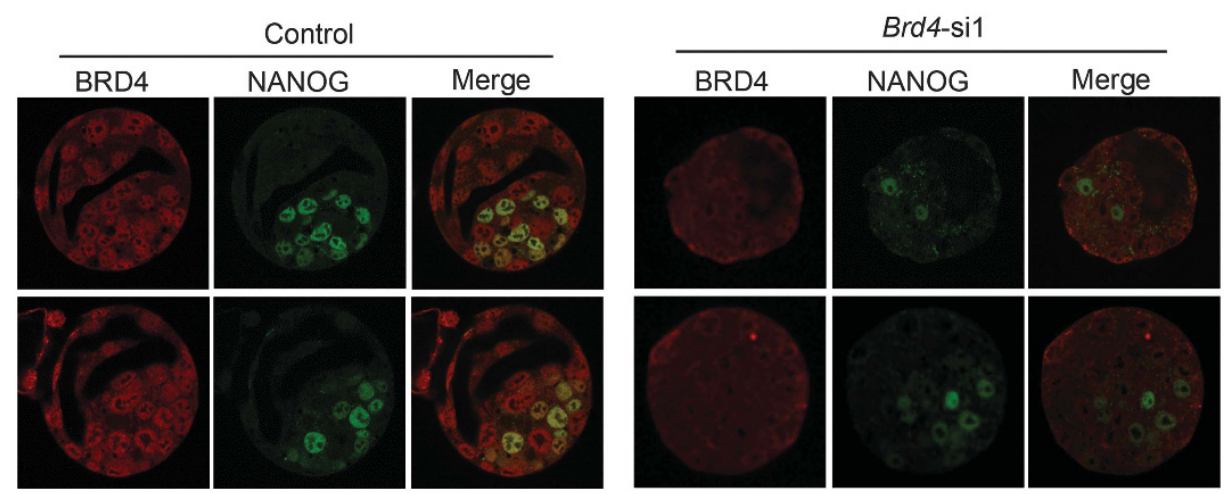

Figure 4 Brd4 knockdown reduces NANOG levels in preimplantation embryos. Two-cell embryos were microinjected with control siRNA or Brd4 siRNA 1 and cultured to the late morula (a) or blastocyst (b) stage. Immunofluorescence was performed for BRD4 (red) and NANOG (green). The experiment was performed three times using at least 20 embryos per group. Representative images are shown

using an affinity-purified Brd4 antibody. ${ }^{25}$ The proximal promoter $(-188)$ and distal enhancer $(-4546)$ regions of Nanog gene have been defined previously. ${ }^{26-28}$ Primers spanning these regions were used in ChIP-qPCR assays to detect the binding of BRD4 (Figure 5a). Based on a published study, ${ }^{29}$ we also designed primer pairs for two additional Nanog regulatory regions ( -951 and -3329$)$. In addition, a pair of primers targeting a region $\sim 6000$-bp downstream of Nanog transcription start site was used to show Brd4 binding outside the Nanog gene (Figure 5a, $+6001)$.

As JQ1(+) promotes BRD4 dissociation from chromatin, we tested how it affects BRD4 binding to the Nanog locus. BRD4 ChIPs were performed for both TL1 and R1 cells treated with $500 \mathrm{nM}$ of either JQ1(+) or JQ1(-) for $6 \mathrm{~h}$, at which time JQ1 $(+)$ does not have a significant effect on cell proliferation (Supplementary Figure S8). In JQ1( - )-treated cells, BRD4 was specifically enriched at the previously established Nanog core proximal promoter and distal enhancer regions (Figure $5 b$ and Supplementary Figure S8A). In contrast, BRD4 binding at the +6001 region was much lower in both TL1 and R1 cells (Figure $5 \mathrm{~b}$ and Supplementary Figure S9). JQ1 (+) treatment efficiently inhibited BRD4 binding to the Nanog promoter and enhancer regions (Figure $5 \mathrm{~b}$ and Supplementary Figure S9). These data support the observation that JQ1 ( + ) inhibits Nanog expression in ESCs (Figure 2 and Supplementary Figure S1) and further demonstrate that Brd4 is recruited to the Nanog promoter and enhancer regions to support Nanog expression, thereby identifying Nanog as a novel BRD4 target gene in ESCs. 
a
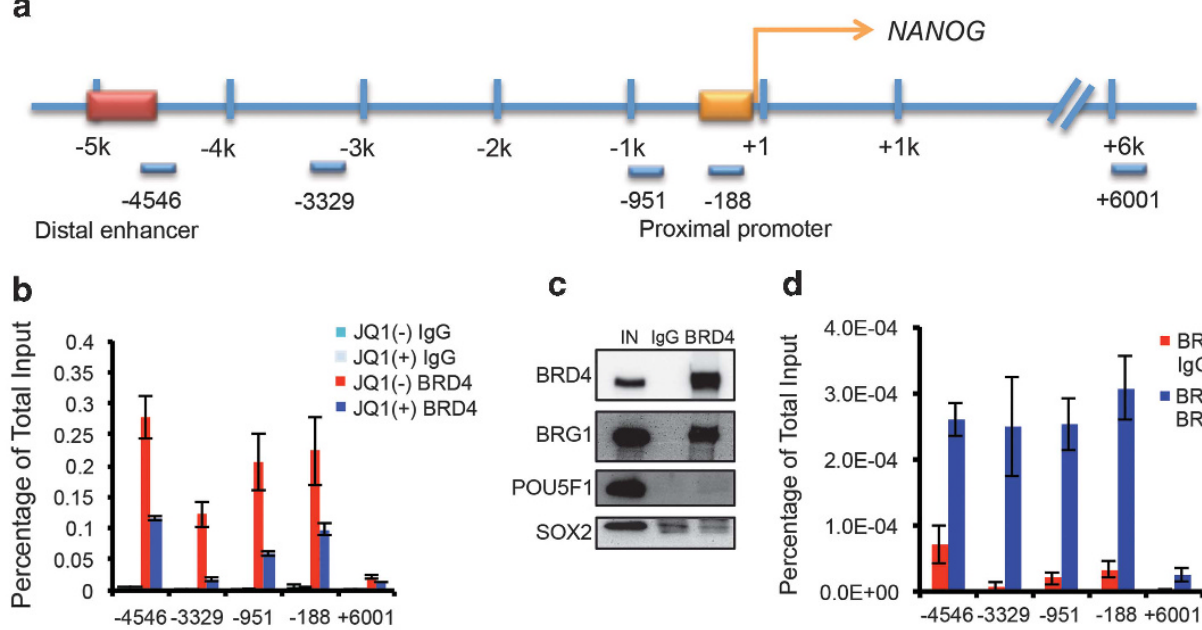

c

d
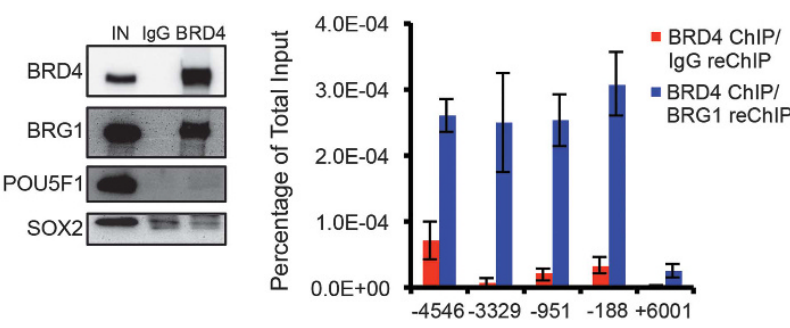

e
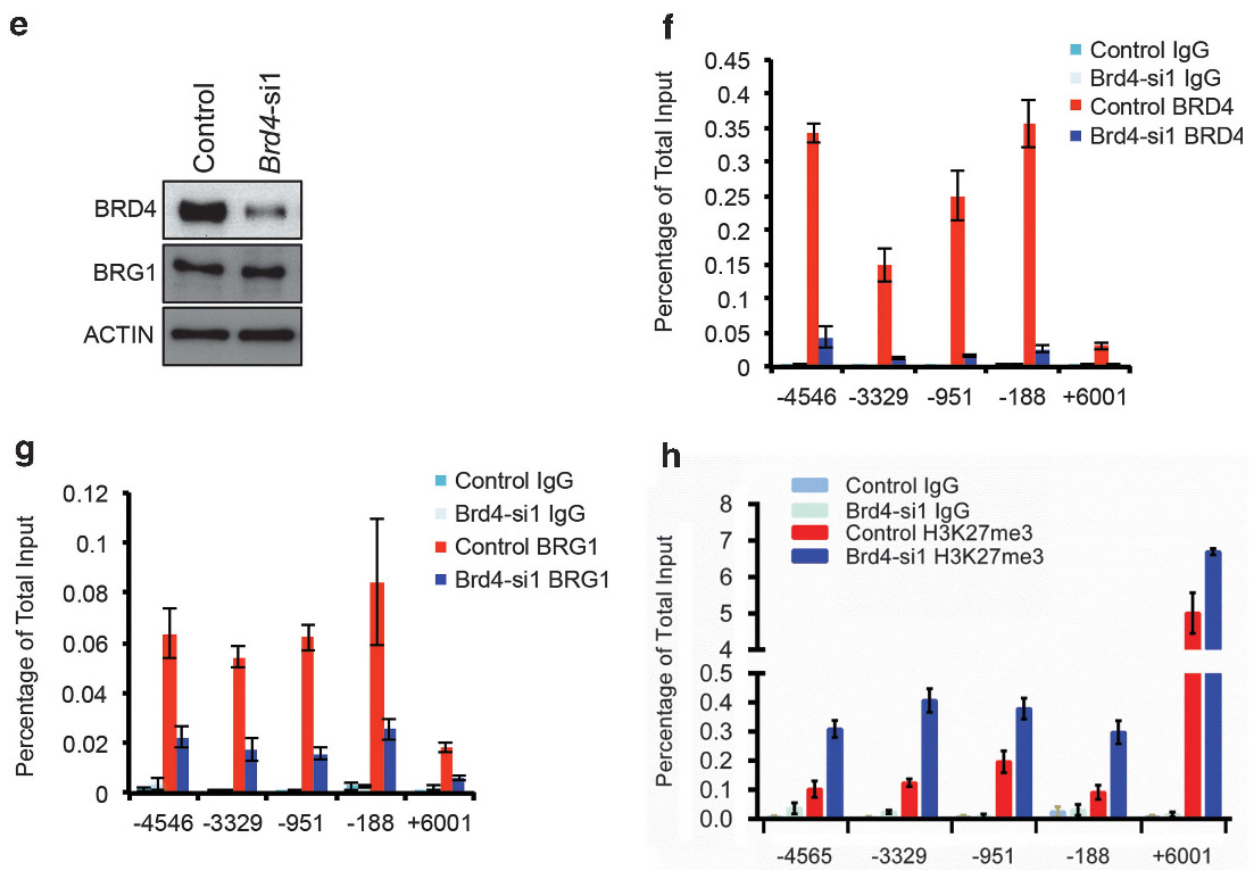

Figure 5 BRD4 interacts with BRG1 in the Nanog regulatory regions to regulate its expression in ESCs. (a) Schematic diagram of the Nanog promoter showing regions analyzed in ChIP. (b) R1 cells were treated with $500 \mathrm{nM} \mathrm{JQ1}-$ ) or JQ1 ( ) for $6 \mathrm{~h}$ and subjected to the ChIP assay with normal rabbit IgG or BRD4 antibody. ChIP samples were analyzed by qPCR using primers targeting the regions indicated in a. (c) R1 nuclear extracts immunoprecipitated with normal rabbit IgG or the BRD4 antibody were immunoblotted with the indicated antibodies. IN, input. (d) For the ChIP-reChIP assays using R1 ESCs, the primary ChIP with BRD4C antibody was followed by a secondary ChIP (reChIP) using either IgG or a BRG1 antibody. (e) R1 ESCs were transfected with control siRNA or Brd4 siRNA 1. At 48-h post-transfection, whole-cell extracts were extracted and immunoblotted with the indicated antibodies. ACTIN was used as a loading control. (f-h) R1 ESCs were transfected as in e. At 48-h post-transfection, the cells were subjected to ChIP assay with normal rabbit IgG, BRD4C antibody (f), BRG1 antibody (g) or H3K27me3 antibody (h). All ChIP values represent the average of three independent experiments with error bars indicating S.E.M.

\section{BRD4 regulates Nanog expression through association} with BRG1. We further examined the molecular mechanism by which BRD4 regulates Nanog expression. In mouse ESCs, BRG1, which is the ATPase subunit of an ESCspecific chromatin-remodeling complex, ${ }^{30}$ has critical roles in transcriptional regulation of key factors in ESC self-renewal and pluripotency. ${ }^{30,31}$ We identified BRG1 as a BRD4interacting protein by mass spectrometry (Supplementary Figure S10A). As BRG1 binds to the Nanog promoter to support gene expression, ${ }^{30,31}$ we speculated that BRD4 may regulate Nanog expression through interaction with BRG1. We then performed co-immunoprecipitation to confirm the interaction between BRD4 and BRG1 in ESCs (Figure 5c). The interaction between BRD4 and BRG1 was also validated in an embryonic cancer stem cell line (Supplementary Figure S10B).

BRD4 binds to the same regions in the Nanog locus as those published for BRG1 (Figure 5b, Supplementary Figure S9 and Kidder et al. $^{31}$ ). Accordingly, we tested if BRD4 and BRG1 co-occupy the Nanog regulatory regions in ESCs. 
Sequential ChIP (ChIP-reChIP) showed that BRG1 was specifically enriched with BRD4 in the Nanog regulatory regions (Figure $5 d$ ). We also tested if BRD4 supported BRG1 recruitment to the Nanog regulatory regions. ChIP assays were performed using ESCs transfected with control or Brd4 siRNA to determine how Brd4 knockdown affected BRG1 binding to the Nanog regulatory regions. Western blotting showed that Brd4 knockdown did not affect BRG1 expression (Figure 5e). As expected, binding of BRD4 to the Nanog regulatory regions was greatly reduced following Brd4 knockdown (Figure 5f). More importantly, BRG1 binding to the Nanog regulatory regions was markedly reduced when $\mathrm{Brd} 4$ was knocked down (Figure $5 \mathrm{~g}$ ). This result suggests that BRD4 is important for recruiting BRG1 to their common binding sites at the Nanog locus. It is also possible that inhibition of Nanog expression by Brd4 knockdown reduces BRG1 recruitment to the Nanog regulatory regions through other mechanism(s).

BRG1 activates pluripotent gene expression by binding to the target genes and preventing localized trimethylation of Lys27 of histone 3 (H3K27me3), which is commonly associated with gene repression. ${ }^{30}$ This BRG1 function prompted us to test how Brd4 knockdown-mediated inhibition of BRG1 recruitment to the Nanog regulatory regions affects H3K27me3 modifications at this site. ChIP assays showed that in control cells the repressive H3K27me3 modification was present at the Nanog regulatory regions at relatively low levels compared with the +6001 region (Figure $5 \mathrm{~h}$ ). Interestingly, the higher level of $\mathrm{H} 3 \mathrm{~K} 27 \mathrm{me} 3$ at the +6001 region was inversely correlated with the low level of BRD4 and BRG1 detected in this region. Brd4 knockdown led to an increase of H3K27me3 at the Nanog regulatory regions (Figure 5h). As BRG1 has been shown to repress H3K27me3 silencing modifications at its target genes genome wide, ${ }^{30}$ our observations suggest that when BRG1 recruitment was inhibited by Brd4 knockdown, H3K27me3 expanded into the Nanog regulatory regions, leading to formation of repressive chromatin and transcriptional silencing. Collectively, these studies suggest that BRD4 associates with BRG1 chromatinremodeling protein in the Nanog regulatory regions to inhibit H3K27me3 silencing modifications, thereby promoting Nanog expression in ESCs.

\section{Discussion}

We report here that the amount of BRD4 protein is markedly decreased during mouse ESC differentiation, suggesting that BRD4 has an important role in undifferentiated ESCs. We further demonstrate that BRD4 specifically regulates the pluripotency transcription factor Nanog in ESCs. BRD4 inhibitors and Brd4 knockdown cause great inhibition of Nanog expression, leading to impaired ESC maintenance. More importantly, Brd4 knockdown markedly inhibits Nanog expression in the late morula and blastocyst stage embryos. Together, these studies demonstrate that BRD4 is critical for regulating Nanog expression both in ESCs and during preimplantation development.

In mouse embryos, NANOG functions primarily during formation and expansion of the ICM. ${ }^{16}$ Nanog-null embryos are not able to establish a pluripotent ground state because
Nanog-deficient ICMs do not generate pluripotent cells. ${ }^{14}$ BRD4's role in Nanog gene expression unmasked in this study therefore provides an explanation for ICM degeneration in Brd4 knockout mice, ${ }^{10}$ revealing a novel function for $\mathrm{Brd} 4$ in preimplantation embryo development.

In addition to maintaining pluripotency in ESCs, Nanog overexpression is observed in a large number of cancers, where it not only correlates with a poor clinical outcome but also regulates cancer stem cell sub-populations. ${ }^{32} \mathrm{We}$ observe BRD4 binding to Nanog regulatory regions and regulation of Nanog expression in two embryonic cancer stem cell lines, P19 and NTERA-2 (Supplementary Figure S11), suggesting that this function of BRD4 could be preserved during cancer development. This finding is important in light of recent studies revealing that stem cell regulatory networks frequently contribute to the ESC-like phenotype displayed by many aggressive tumors. ${ }^{33,34}$

We also find that BRD4 is recruited to Nanog promoter and enhancer regions and this occupancy is inhibited by either JQ1(+) or Brd4 knockdown. These results are consistent with recent studies showing that Brd4 binds to the 'superenhancer' regions of key cell identity genes. ${ }^{3,35}$ We find that BRD4 complexes with BRG1 at the Nanog locus and inhibits H3K27me3 silencing modifications to promote Nanog expression. Currently, very little is known about how BRD4 function is coupled to chromatin remodeling besides the fact that it binds to acetylated histones. The interplay between BRD4 and the epigenetic reprogramming factor BRG1 in regulating Nanog expression described here provides new insights for understanding the epigenetic event that modulates BRD4 function in ESCs. In line with our finding, BRD4 and BRG1 were recently reported to be enriched in the same regions of superenhancers in ESCs. ${ }^{9}$ As a result the BRG1-associated SWI/ SNF chromatin-remodeling complex is the most frequently mutated chromatin-regulatory complex in human cancer, ${ }^{36}$ it will be important to determine if the tumor suppression function of the BRG1 complex is abrogated in BRD4associated cancers.

In summary, this study identifies Nanog as a novel target gene regulated by BRD4. The functional interaction between BRD4 and BRG1 provides novel insights for the mechanism by which BRD4 functions in transcription regulation as well as the molecular event that regulates Nanog expression in ESCs. Many epigenetic readers such as BRD4 recognize various histone modifications, and translate the 'histone codes' into gene expression/repression, providing a tight control for cell proliferation. Dysfunction of this process frequently leads to cancers and other human diseases, underscoring the biological significance of epigenetic signaling. By elucidating the biological function of the important cancer target BRD4, our findings lay the groundwork for understanding how dysregulation of BRD4 function could contribute to cancer development.

\section{Materials and Methods}

Cell culture. Mouse TL1 and R1 ESCs (kindly provided by Dr. Mitchell $J$ Weiss, the Children's Hospital of Philadelphia, Philadelphia, PA, USA) were maintained feeder-free on gelatin-coated tissue culture dishes in Knockout DMEM/ F12 medium (Invitrogen, Life Technologies, Grand Island, NY, USA) with $0.5 \%$ $\mathrm{N}-2$ supplement (Invitrogen), 1\% B27 supplement without vitamin A (Invitrogen), 
0.05\% BSA (Invitrogen), 1\% GlutaMAX (Invitrogen), $10 \mathrm{ng} / \mathrm{ml}$ human BMP4 (R\&D, Minneapolis, MN, USA), $1000 \mathrm{U} / \mathrm{ml}$ LIF (Millipore, Bedford, MA, USA), $1.5 \times 10^{-4} \mathrm{M}$ 1-thioglycerol (Sigma, St. Louis, MO, USA), $100 \mathrm{nM}$ FGF receptor inhibitor PD173074 (Sigma) and 1\% Pen-Strep as described previously. ${ }^{15,37,38}$ For the 2i/LIF condition, ESCs were also maintained feeder-free on gelatin-coated tissue culture dishes in Knockout DMEM/F12 medium (Invitrogen) with $0.5 \% \mathrm{~N}-2$ supplement (Invitrogen), 1\% B27 supplement without vitamin A (Invitrogen), 0.05\% BSA (Invitrogen), 1\% GlutaMAX (Invitrogen), $1.5 \times 10^{-4} \mathrm{M}$ 1-thioglycerol (Sigma), $1000 \mathrm{U} / \mathrm{ml}$ LIF (Millipore), $1 \mu \mathrm{M}$ PD0325901 and $3 \mu \mathrm{M}$ CHIR99021 as described previously. ${ }^{39}$ The ESCs were cultured on gelatin-coated plates and propagated by trypsinization and re-plating at a ratio of 1 to 10 every 2 or 3 days. All cell lines were depleted of feeder cells and transitioned to serum-free medium over several passages before experiments as described. ${ }^{40}$ HEK $293 \mathrm{~T}$ cells were maintained in Dulbecco's modified Eagle's medium (Invitrogen) with 10\% fetal calf serum (FBS) (Hyclone, Logan, UT, USA) or 10\% Tet system approved FBS (Clontech, Mountain View, CA, USA). P19 Cells (kindly provided by Dr. M Celeste Simon, University of Pennsylvania, Philadelphia, PA, USA) were maintained in aMEM medium (Invitrogen) with $7.5 \%$ newborn calf serum (Hyclone) and $2.5 \%$ FBS (Hyclone).

siRNA knockdown. The non-targeting control siRNA (D-001210-01), Brd4 siRNA 1 (D-004937-02) and Brd4 siRNA 2 (D-004937-04) were purchased from Dharmacon (Thermo Scientific, Hudson, NH, USA). For siRNA-mediated knockdown, ESCs were transfected during subculture using DharmaFECT 2 (Thermo Scientific) following the manufacturer's instructions. At 18- to 24-h post-transfection, the medium was changed to ESC medium without FGF receptor inhibitor PD173074 (Sigma). P19 cells were transfected during subculture using DharmaFECT 3 (Thermo Scientific). All transfections were performed following the manufacturer's instructions.

Retinoic-acid-induced differentiation. ESCs were trypsinized and plated on gelatin-coated plates in ESC medium. The next day, the medium was replaced with ESC medium without LIF and BMP4 but with $1 \mu \mathrm{M}$ RA (Sigma). Cells were cultured for an additional 48 or $96 \mathrm{~h}$ with one medium change.

Colony formation assays. For JQ1 $(+)$ experiment, ESCs were trypsinized to obtain a single-cell suspension and plated at 150000 cells per well in six-well plate using Knockout DMEM/F12 medium (Invitrogen) with $0.5 \% \mathrm{~N}-2$ supplement (Invitrogen), 1\% B27 supplement without vitamin A (Invitrogen), 0.05\% BSA (Invitrogen), 1\% GlutaMAX (Invitrogen), $10 \mathrm{ng} / \mathrm{ml}$ human BMP4 (R\&D), $1000 \mathrm{U} / \mathrm{ml} \mathrm{LIF}$ (Millipore) and $1.5 \times 10^{-4} \mathrm{M} 1$-thioglycerol (Sigma). After 2 days of culture, plates were stained for AP using the Alkaline Phosphatase Staining Kit (Sigma) following the manufacturer's recommendation. For Brd4 knockdown experiments, siRNA-transfected cells were seeded at 500 cells per well in 12-well plates. The AP staining was performed after 6 days of culture.

Immunoprecipitation and western blot analysis. These analyses were performed using standard protocols. Additional experimental details are provided in Supplementary Information Materials and Methods.

ESC immunofluorescent staining. Cells cultured on chamber slides were fixed with $3 \%$ paraformaldehyde in PBS for $20 \mathrm{~min}$. Immunofluorescent staining was performed as previously described. ${ }^{41}$ The following primary antibodies were used: anti-BRD4C (recognizes BRD4 aa 1313-1362, $1: 20000)$, anti-SOX2 (1:1000, MAB2018, R\&D), anti-NANOG $(1: 1000$, ab80892, Abcam, Cambridge, UK), anti-Nanog (1:1000, 560259, BD Pharmingen, San Diego, CA, USA) and anti-Xpress (1:1000, R910-25, Invitrogen). The secondary antibodies used were Alexa Fluor 594 goat anti-rabbit IgG $(1: 1000$, A11012, Invitrogen) and Alexa Fluor 488 goat anti-mouse IgG (1:500, A11001, Invitrogen). All immunofluorescent images were captured using an inverted fluorescence microscope (Olympus, IX81, Tokyo, Japan) as described previously. ${ }^{22}$ The scale bars were added using ImageJ software (National Institutes of Health, Bethesda, MD, USA).

Quantitative reverse transcription and real-time PCR. Total RNA was isolated using NucleoSpin RNA II Kit (Macherey-Nagel, Dueren, Germany) following the manufacturer's instruction. Reverse transcription was performed using a $20 \mu$ reaction mixture containing $350 \mathrm{ng}$ of total RNA, oligo(dT) primer (Invitrogen), dNTP (Invitrogen) and M-MLV reverse transcriptase (Invitrogen).
Quantitative real-time PCR was performed using a CFX96 real-time PCR detection system (Bio-Rad, Hercules, CA, USA) with IQ SYBR Green supermix (Bio-Rad). mRNA level of each gene was normalized to Gapdh mRNA level. Primer sequences are shown in Supplementary Table S2.

ChIP and ChIP-reChIP. ChIP assay using TL1, R1 or P19 cells were performed based on published methods with the modifications indicated below. ${ }^{42,43}$ Formaldehyde was added directly to cell culture media at a final concentration of $1 \%$. Fixation was completed after incubation for $10 \mathrm{~min}$ at room temperature and stopped by adding glycine to a final concentration of $0.125 \mathrm{M}$. Cells were scraped, collected, centrifuged and swelled in cell lysis buffer $(5 \mathrm{mM}$ PIPES, pH 8.0, $85 \mathrm{mM} \mathrm{KCl,} 1 \% \mathrm{NP}-40,0.1 \mathrm{mM}$ PMSF and $1 \mu \mathrm{g} / \mathrm{ml}$ leupeptin, aprotinin and pepstatin A). After 1-h incubation on ice, nuclei were collected by centrifugation at $3200 \mathrm{~g}$ for $10 \mathrm{~min}$ in $4{ }^{\circ} \mathrm{C}$, resuspended in nuclei lysis buffer (50 mM Tris-HCl, pH 8.0, 1\% SDS, $10 \mathrm{mM}$ EDTA, $0.1 \mathrm{mM} \mathrm{PMSF}$, and $1 \mu \mathrm{g} / \mathrm{ml}$ leupeptin, aprotinin and pepstatin A), and incubated on ice for $10 \mathrm{~min}$. Samples were sonicated on ice to an average of 500-bp DNA length and centrifuged at $20000 \mathrm{~g}$. The chromatin solution was pre-cleared with Staph A cells (pre-blocked with $1 \mathrm{mg} / \mathrm{ml}$ sheared herring sperm DNA and $1 \mathrm{mg} / \mathrm{ml} \mathrm{BSA}$ at $4{ }^{\circ} \mathrm{C}$ overnight) for $15 \mathrm{~min}$ at $4{ }^{\circ} \mathrm{C}$. Chromatin from about $10^{7}$ cells was incubated with $3 \mu \mathrm{g}$ of normal rabbit IgG (Upstate, Millipore), an affinity-purified rabbit polyclonal antibody generated in our lab for BRD4C (recognizes BRD4 aa 1313-1362), an anti-Brg1 antibody (J1, kindly provided by Dr. Weidong Wang, National Institute on Aging, $\mathrm{NIH}$, Baltimore, MD, USA) or H3K27me3 antibody (07-449, Millipore). After rotating at $4{ }^{\circ} \mathrm{C}$ overnight, chromatin and antibody complexes were immunoprecipitated by mixing with pre-blocked Staph $\mathrm{A}$ cells at $4^{\circ} \mathrm{C}$ for $15 \mathrm{~min}$. Immunoprecipitates were centrifuged at $20000 \mathrm{~g}$ for $5 \mathrm{~min}$. The supernatant from the normal rabbit IgG antibody sample was saved as total chromatin input. Staph A immuno-complexes were washed twice with dialysis buffer $(50 \mathrm{mM}$ Tris- $\mathrm{HCl}, \mathrm{pH}$ 8.0, $2 \mathrm{mM}$ EDTA, $0.2 \%$ Sarkosyl and 0.1 mM PMSF) and four times with ChIP wash buffer (100 mM Tris, pH 9.0, $500 \mathrm{mM} \mathrm{LiCl,} \mathrm{1 \%} \mathrm{NP-40,1 \%} \mathrm{deoxycholic} \mathrm{acid}$ and $0.1 \mathrm{mM}$ PMSF). Immunocomplexes were eluted from the Staph A cells using elution buffer ( $50 \mathrm{mM} \mathrm{NaHCO}$ and $1 \%$ SDS). Crosslinks were reversed by adding $\mathrm{NaCl}$ to the eluted supernatants to a final concentration of $300 \mathrm{mM}$ and incubating at $67^{\circ} \mathrm{C}$ overnight. RNA was removed by incubation with RNase $\mathrm{A}$ at $37^{\circ} \mathrm{C}$ for $30 \mathrm{~min}$. DNA samples were purified using PCR purification kit (Qiagen, Gaithersburg, MD, USA) and eluted in $50 \mu \mathrm{l}$ EB buffer. Real-time PCR was performed as described above using primers listed in Supplementary Table S2. Two micro-liters of IPed DNA or 1/50 of total input chromatin was used as templates for PCR reaction.

For ChIP-reChIP assay, the first ChIP was performed using BRD4C antibody as described above. For the reChIP experiments, protein-DNA complexes isolated from the first ChIP were washed with dialysis buffer and washing buffer described in the ChIP protocol. Complexes were eluted by incubation with elution buffer $(50 \mathrm{mM}$ Tris-Cl pH 8.0 and $50 \mathrm{mM}$ EDTA supplemented with 1\% SDS and protease inhibitors) for $15 \mathrm{~min}$ at $65^{\circ} \mathrm{C}$. After centrifugation, the supernatants were diluted 10 times with ChIP dilution buffer supplemented with $20 \mu \mathrm{g}$ GST-BRD4CTD described above, $50 \mu \mathrm{g}$ of BSA and protease inhibitors. After rotating at $4{ }^{\circ} \mathrm{C}$ for $2 \mathrm{~h}$, the chromatin solution was pre-cleared with Staph $A$ cells for $15 \mathrm{~min}$ at $4{ }^{\circ} \mathrm{C}$ and centrifuged at $20000 \mathrm{~g}$ for $5 \mathrm{~min}$ at $4{ }^{\circ} \mathrm{C}$. The supernatants were then subjected to the second ChIP procedure.

Oocyte and embryo collection, culture and microinjection. Fullgrown germinal vesicle (GV)-intact oocytes, metaphase II (MII)-arrested eggs and preimplantation embryos were collected from 6- to 8-week-old CF1 female mice (Harlan, Indianapolis, IN, USA). GV oocytes were collected from mice $46-48 \mathrm{~h}$ after injection with $5 \mathrm{IU}$ eCG. For MII-egg and embryo collection, female mice were injected with 5 IU eCG followed by 5 IU hCG $46-48 \mathrm{~h}$ later. MII eggs were obtained from the oviducts $13-15 \mathrm{~h}$ after hCG injection. For embryo collection, after hCG administration, females were mated to B6D2F1/J males (Jackson Laboratory, Bar Harbor, ME, USA). One-cell, two-cell, four-cell, eight-cell and blastocyst stage embryos were flushed from the oviducts/uteri of superovulated and mated mice 20,44, 61, 68 and 96-h post-hCG, respectively. The collection medium was bicarbonate-free minimal essential medium (Earle's salt) supplemented with $3 \mathrm{mg} / \mathrm{ml}$ of polyvinylpyrrolidone (PVP) and $25 \mathrm{mM}$ Hepes (MEM/PVP), pH 7.3. For oocyte collection, $2.5 \mu \mathrm{M}$ milrinone (Sigma-Aldrich, St. Louis, MO, USA) was added to the collection medium to prevent spontaneous resumption of meiosis. For the microinjection experiments, two-cell embryos were collected and cultured in KSOM medium containing amino acids 
$(\mathrm{KSOM}+\mathrm{AA})^{44}$ at $37^{\circ} \mathrm{C}$ in a humidified atmosphere containing $5 \% \mathrm{CO}_{2}$ in air. One of the two blastomeres of a two-cell embryo was microinjected in MEM/PVP with $\sim 5 \mu \mathrm{l}$ of $20 \mu \mathrm{M}$ non-target control siRNA (D-001210-01) or Brd4 siRNA1 (D-004937-02). The injections were performed as previously described. ${ }^{45}$ Microinjected embryos were returned to KSOM + AA and cultured for 2 days (morula) or 3 days (blastocyst) at $37^{\circ} \mathrm{C}$

Embryo immunofluorescent staining and laser-scanning confocal microscopy. Immunofluorescence was performed as previously described, ${ }^{46}$ except that the zona pellucida was not removed. Both anti-BRD4C (recognizes BRD4 aa 1313-1362) and anti-Nanog (560259, BD Pharmingen) antibodies were used at a 1:100 dilution. Anti-Pou5f1 antibody (sc-8628, Santa Cruz, Santa Cruz, CA, USA) was used at a 1:50 dilution. FITC-conjugated goat anti-mouse IgG1 (SouthernBiotech, Birmingham, $A L, U S A), C y 5-c o n j u g a t e d$ donkey anti-rabbit IgG and anti-goat IgG (both from Jackson ImmunoResearch, West Grove, PA, USA), all at a 1:100 dilution, were used as secondary antibodies. DNA was stained with $1 \mu \mathrm{M}$ SYTOX Green (Life Technologies). Fluorescence was detected on a Leica TCS SP laser-scanning confocal microscope (Wetzlar, Germany). For each experiment, all images were taken at the same laser power and single confocal sections were collected.

JQ1 treatment of human ESCs. The H9 human ESC line was obtained from WiCell Research Institute (Madison, WI, USA) and was cultured feeder-free under standardized conditions in a humidified incubator at $37^{\circ} \mathrm{C} / 5 \% \mathrm{CO}_{2} / 5 \% \mathrm{O}_{2}$. Cells were plated onto Matrigel (BD Biosciences, San Diego, CA, USA) coated plates and grown in mTeSR 1 medium (Stemcell Technologies, Vancouver, BC, Canada). Cells were passaged every 4-6 days using Accutase (Innovative Cell Technologies, San Diego, CA, USA). For JQ1 treatment, H9 cells were passaged into six-well plates and grown for 2-3 days until cells were exponentially growing and small colonies were visible. The cells were then treated with $300 \mathrm{nM} \mathrm{JQ1}(+)$ or $\mathrm{JQ1}(-)$ in $\mathrm{mTeSR}^{\mathrm{TM}_{1}}$ media for $48 \mathrm{~h}$. Untreated cells were used as a control. Media containing fresh compounds were replaced daily. After $48 \mathrm{~h}$, cells were washed once in $\mathrm{mTeSR}^{\mathrm{TM}} 1$ media and harvested for western blot analysis.

Ethics statement. All procedures involving animals have been conducted as approved by the University of Pennsylvania Institutional Animal Care and Use Committee.

\section{Conflict of Interest}

The authors declare no conflict of interest.

Acknowledgements. We thank M Celeste Simon (University of Pennsylvania) for P19 cells, Mitchell J Weiss (The Children's Hospital of Philadelphia) for R1 cells, James E Bradner (Dana-Farber Cancer Institute) for JQ1, Weidong Wang $(\mathrm{NIH})$ for Brg1 antibody and Jing Jiao for technical support. We thank members of our laboratories for helpful discussion and critical review of the manuscript. This work has been supported by $\mathrm{NIH}$ grants R01CA148768 and R01CA142723 to JY and R01HD022681 to RMS.

1. Dey A, Ellenberg J, Farina A, Coleman AE, Maruyama T, Sciortino S et al. A bromodomain protein, MCAP, associates with mitotic chromosomes and affects $\mathrm{G}(2)$-to-M transition. $\mathrm{Mol}$ Cell Biol 2000; 20: 6537-6549.

2. Wu SY, Chiang CM. The double bromodomain-containing chromatin adaptor Brd4 and transcriptional regulation. J Biol Chem 2007; 282: 13141-13145.

3. Loven J, Hoke HA, Lin CY, Lau A, Orlando DA, Vakoc CR et al. Selective inhibition of tumor oncogenes by disruption of super-enhancers. Cell 2013; 153: 320-334.

4. Zuber J, Shi J, Wang E, Rappaport AR, Herrmann H, Sison EA et al. RNAi screen identifies Brd4 as a therapeutic target in acute myeloid leukaemia. Nature 2011; 478 524-528.

5. Rodriguez RM, Huidobro C, Urdinguio RG, Mangas C, Soldevilla B, Dominguez G et al. Aberrant epigenetic regulation of bromodomain BRD4 in human colon cancer. $\mathrm{J} \mathrm{Mol} \mathrm{Med}$ (Berl) 2012; 90: 587-595

6. Filippakopoulos $P, Q i$ J, Picaud S, Shen Y, Smith WB, Fedorov $O$ et al. Selective inhibition of BET bromodomains. Nature 2010; 468: 1067-1073.

7. Crawford NP, Alsarraj J, Lukes L, Walker RC, Officewala JS, Yang HH et al. Bromodomain 4 activation predicts breast cancer survival. Proc Natl Acad Sci USA 2008; 105 6380-6385.
8. French CA, Miyoshi I, Aster JC, Kubonishi I, Kroll TG, Dal Cin P et al. BRD4 bromodomain gene rearrangement in aggressive carcinoma with translocation $\mathrm{t}(15 ; 19)$. Am $\mathrm{J}$ Pathol 2001; 159: 1987-1992.

9. Hnisz D, Abraham BJ, Lee TI, Lau A, Saint-Andre V, Sigova AA et al. Super-Enhancers in the Control of Cell Identity and Disease. Cell 2013; 155: 934-947.

10. Houzelstein D, Bullock SL, Lynch DE, Grigorieva EF, Wilson VA, Beddington RS. Growth and early postimplantation defects in mice deficient for the bromodomain-containing protein Brd4. Mol Cell Biol 2002; 22: 3794-3802.

11. Nishiyama A, Dey A, Miyazaki J, Ozato K. Brd4 is required for recovery from antimicrotubule drug-induced mitotic arrest: preservation of acetylated chromatin. Mol Biol Cell 2006; 17: 814-823.

12. Boyer LA, Lee TI, Cole MF, Johnstone SE, Levine SS, Zucker JP et al. Core transcriptional regulatory circuitry in human embryonic stem cells. Cell 2005; 122: 947-956.

13. Young RA. Control of the embryonic stem cell state. Cell 2011; 144: 940-954.

14. Silva J, Nichols J, Theunissen TW, Guo G, van Oosten AL, Barrandon O et al. Nanog is the gateway to the pluripotent ground state. Cell 2009; 138: 722-737

15. Mitsui K, Tokuzawa $\mathrm{Y}$, Itoh $\mathrm{H}$, Segawa K, Murakami M, Takahashi $\mathrm{K}$ et al. The homeoprotein Nanog is required for maintenance of pluripotency in mouse epiblast and ES cells. Cell 2003; 113: 631-642.

16. Chambers I, Silva J, Colby D, Nichols J, Nijmeijer B, Robertson $M$ et al. Nanog safeguards pluripotency and mediates germline development. Nature 2007; 450: 1230-1234

17. Ivanova N, Dobrin R, Lu R, Kotenko I, Levorse J, DeCoste $\mathrm{C}$ et al. Dissecting self-renewal in stem cells with RNA interference. Nature 2006; 442: 533-538.

18. Nichols J, Zevnik B, Anastassiadis $\mathrm{K}$, Niwa $\mathrm{H}$, Klewe-Nebenius $\mathrm{D}$, Chambers I et al. Formation of pluripotent stem cells in the mammalian embryo depends on the POU transcription factor Oct4. Cell 1998; 95: 379-391.

19. Do DV, Ueda J, Messerschmidt DM, Lorthongpanich C, Zhou Y, Feng B et al. A genetic and developmental pathway from STAT3 to the OCT4-NANOG circuit is essential for maintenance of ICM lineages in vivo. Genes Dev 2013; 27: 1378-1390.

20. Davis AC, Wims M, Spotts GD, Hann SR, Bradley A. A null c-myc mutation causes lethality before 10.5 days of gestation in homozygotes and reduced fertility in heterozygous female mice. Genes Dev 1993; 7: 671-682

21. Nishiyama A, Sharov AA, Piao $Y$, Amano M, Amano T, Hoang HG et al. Systematic repression of transcription factors reveals limited patterns of gene expression changes in ES cells. Sci Rep 2013; 3: 1390.

22. Wang R, Li Q, Helfer CM, Jiao J, You J. Bromodomain protein Brd4 associated with acetylated chromatin is important for maintenance of higher-order chromatin structure. J Biol Chem 2012; 287: 10738-10752.

23. Miyanari Y, Torres-Padilla ME. Control of ground-state pluripotency by allelic regulation of Nanog. Nature 2012; 483: 470-473

24. Nagashima T, Maruyama T, Furuya M, Kajitani $\mathrm{T}$, Uchida $\mathrm{H}$, Masuda $\mathrm{H}$ et al. Histone acetylation and subcellular localization of chromosomal protein BRD4 during mouse oocyte meiosis and mitosis. Mol Hum Reprod 2007; 13: 141-148.

25. Yan J, Diaz J, Jiao J, Wang R, You J. Perturbation of BRD4 protein function by BRD4-NUT protein abrogates cellular differentiation in NUT midline carcinoma. J Biol Chem 2011; 286: 27663-27675

26. Jiang J, Chan YS, Loh YH, Cai J, Tong GQ, Lim CA et al. A core Klf circuitry regulates self-renewal of embryonic stem cells. Nat Cell Biol 2008; 10: 353-360.

27. Kuroda T, Tada M, Kubota H, Kimura H, Hatano SY, Suemori $\mathrm{H}$ et al. Octamer and Sox elements are required for transcriptional cis regulation of Nanog gene expression. Mol Cell Biol 2005; 25: 2475-2485.

28. Rodda DJ, Chew JL, Lim LH, Loh YH, Wang B, Ng HH et al. Transcriptional regulation of nanog by OCT4 and SOX2. J Biol Chem 2005; 280: 24731-24737.

29. Ito S, D'Alessio AC, Taranova OV, Hong K, Sowers LC, Zhang Y. Role of Tet proteins in $5 \mathrm{mC}$ to $5 \mathrm{hmC}$ conversion, ES-cell self-renewal and inner cell mass specification. Nature 2010; 466: 1129-1133.

30. Ho L, Miller EL, Ronan JL, Ho WQ, Jothi R, Crabtree GR. esBAF facilitates pluripotency by conditioning the genome for LIF/STAT3 signalling and by regulating polycomb function. Nat Cell Biol 2011; 13: 903-913.

31. Kidder BL, Palmer S, Knott JG. SWI/SNF-Brg1 regulates self-renewal and occupies core pluripotency-related genes in embryonic stem cells. Stem Cells 2009; 27: 317-328.

32. Jeter CR, Badeaux M, Choy G, Chandra D, Patrawala L, Liu C et al. Functional evidence that the self-renewal gene NANOG regulates human tumor development. Stem Cells 2009; 27: 993-1005.

33. Reya T, Morrison SJ, Clarke MF, Weissman IL. Stem cells, cancer, and cancer stem cells. Nature 2001; 414: 105-111.

34. Ben-Porath I, Thomson MW, Carey VJ, Ge R, Bell GW, Regev A et al. An embryonic stem cell-like gene expression signature in poorly differentiated aggressive human tumors. Nat Genet 2008; 40: 499-507.

35. Whyte WA, Orlando DA, Hnisz D, Abraham BJ, Lin CY, Kagey MH et al. Master transcription factors and mediator establish super-enhancers at key cell identity genes. Cell 2013; 153: 307-319.

36. Kadoch C, Hargreaves DC, Hodges C, Elias L, Ho L, Ranish J et al. Proteomic and bioinformatic analysis of mammalian SWI/SNF complexes identifies extensive roles in human malignancy. Nat Genet 2013; 45: 592-601. 
37. Ying QL, Nichols J, Chambers I, Smith A. BMP induction of Id proteins suppresses differentiation and sustains embryonic stem cell self-renewal in collaboration with STAT3. Cell 2003; 115: 281-292.

38. Thomson M, Liu SJ, Zou LN, Smith Z, Meissner A, Ramanathan S. Pluripotency factors in embryonic stem cells regulate differentiation into germ layers. Cell 2011; 145 875-889.

39. Ying QL, Wray J, Nichols J, Batlle-Morera L, Doble B, Woodgett J et al. The ground state of embryonic stem cell self-renewal. Nature 2008; 453: 519-523.

40. Ying QL, Smith AG. Defined conditions for neural commitment and differentiation. Methods Enzymol 2003; 365: 327-341.

41. You J, Croyle JL, Nishimura A, Ozato K, Howley PM. Interaction of the bovine papillomavirus E2 protein with Brd4 tethers the viral DNA to host mitotic chromosomes. Cell 2004; 117: 349-360.
42. Boyd KE, Wells J, Gutman J, Bartley SM, Farnham PJ. c-Myc target gene specificity is determined by a post-DNAbinding mechanism. Proc Natl Acad Sci USA 1998; 95 13887-13892.

43. Boyd KE, Farnham PJ. Myc versus USF: discrimination at the cad gene is determined by core promoter elements. Mol Cell Biol 1997; 17: 2529-2537.

44. Ho Y, Wigglesworth K, Eppig JJ, Schultz RM. Preimplantation development of mouse embryos in KSOM: augmentation by amino acids and analysis of gene expression. Mol Reprod Dev 1995; 41: 232-238.

45. Kurasawa S, Schultz RM, Kopf GS. Egg-induced modifications of the zona pellucida of mouse eggs: effects of microinjected inositol 1,4,5-trisphosphate. Dev Biol 1989; 133: 295-304.

46. Anger M, Stein $P$, Schultz RM. CDC6 requirement for spindle formation during maturation of mouse oocytes. Biol Reprod 2005; 72: 188-194.

Supplementary Information accompanies this paper on Cell Death and Differentiation website (http://www.nature.com/cdd) 